\title{
Physical limitations of travel-time-based shallow water tomography
}

\author{
Orlando C. Rodríguez ${ }^{\mathrm{a})}$ and Sérgio M. Jesus \\ Unidade de Ciências Exactas e Humanas, Universidade do Algarve, Campus de Gambelas, 8000_Faro, \\ Portugal
}

(Received 22 November 1999; revised 23 May 2000; accepted 6 September 2000)

\begin{abstract}
Travel-time-based tomography is a classical method for inverting sound-speed perturbations in an arbitrary environment. A linearization procedure enables relating travel-time perturbations to sound-speed perturbations through a kernel matrix. Thus travel-time-based tomography essentially relies on the inversion of the kernel matrix and is commonly called "linear inversion." In practice, its spatial resolution is limited by the number of resolved and independent arrivals, which is a basic linear algebra requirement for linear inversion performance. Physically, arrival independency is much more difficult to determine since it is closely related to the sound propagating channel characteristics. This paper presents a brief review of linear inversion and shows that, in deep water, the number of resolved arrivals is equal to the number of independent arrivals, while in shallow water the number of independent arrivals can be much smaller than the number of resolved arrivals. This implies that in shallow water there are physical limitations to the number of independent travel times. Furthermore, those limitations are explained through the analysis of an equivalent environment with a constant sound speed. The results of this paper are of central importance for the understanding of travel-time-based shallow water tomography. (C) 2000 Acoustical Society of America. [S0001-4966(00)01212-1]
\end{abstract}

PACS numbers: 43.30.Pc, 43.60.Rw [DLB]

\section{INTRODUCTION}

Ocean acoustic tomography has been suggested in the last two decades as a powerful tool for large-scale ocean temperature monitoring. In contrast with standard "local" and "direct" methods, ocean acoustic tomography can be used to remotely determine mean current and temperature evolution through time in an ocean volume bounded by a system of acoustic sources and receivers. ${ }^{1,2}$ Travel-timebased tomography has been widely used in the context of ocean acoustic tomography to invert for sound-speed perturbations of a background (reference) profile. ${ }^{1-5}$ For instance, tomographic inversion can be performed by linearizing the integral relationship between perturbations in travel time and continuous perturbations in sound speed. After linearization, the perturbations in travel time are related to a set of discrete perturbations in sound speed through a kernel matrix, which depends on stable eigenrays of propagation. Sound-speed perturbations can be estimated by calculating a generalized inverse of the kernel matrix and relating back the set of sound-speed perturbations to travel-time perturbations. This technique is sometimes called "linear inversion" and its spatial resolution (i.e., the number of depths at which soundspeed perturbations can be reliably estimated) is fundamentally limited by the number of resolved — and as we will see independent-arrivals.

Despite the significant number of references related to linear inversion most studies are limited to its application in deep water, where the effects of sound reflection on the ocean boundaries can be, to a certain extent, neglected, and

a)Electronic mail: orodrig@ualg.pt acoustic arrivals can be easily resolved for long-range propagation. In shallow water the interaction of sound with the ocean boundaries plays an important role and time resolution of closely spaced arrivals is generally an important practical issue. As an example, Fig. 1 shows a typical shallow water channel impulse response estimate. It is clear from that figure that initial arrivals are unresolved, while late arrivals are well resolved and "clustered" in quadruplets. From raytracing predictions it can be shown that most of the initial unresolved arrivals correspond to refracted and bottom reflected eigenrays, while the quadruplets correspond to surface and bottom reflected eigenrays. An important feature in this example is the significant number of resolved arrivals. In the context of travel-time-based shallow water tomography, and through linear inversion, it seems reasonable that those arrivals should be used to achieve a high spatial resolution of sound-speed estimates. This would be the case providing that all the resolved arrivals are independent, i.e., that all the acoustic arrivals that can be identified from one transmission to another correspond to "pieces" of information independently related to the perturbation of sound speed. This assumption seems to be implicitly accepted in some of the studies concerning linear inversion. ${ }^{1,3,4}$ Nevertheless, it is shown in this paper that for shallow water the number of independent arrivals is in fact smaller, and in some cases much smaller, than the number of actually measuredresolved-arrivals. This result implies that in shallow water part of the acoustic arrivals carry redundant information and therefore there are fundamental physical limitations to the number of independent arrivals. Furthermore, and most importantly, this paper shows that the redundancy of shallow water stable arrivals can be explained through the compari- 


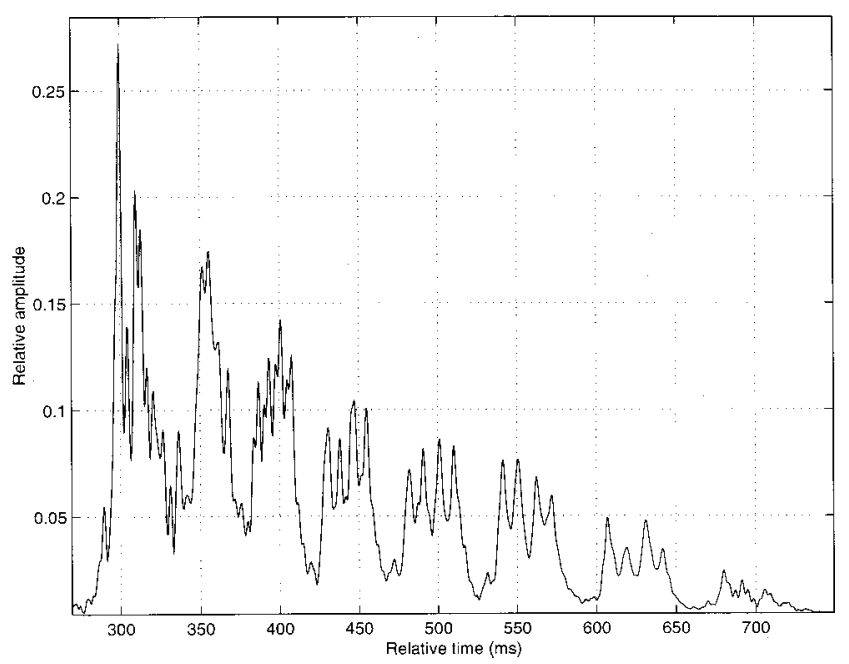

FIG. 1. Typical shallow water short-range arrival pattern showing unresolved (initial) and resolved (late) arrivals; resolved arrivals are "clustered", in groups of quadruplets [real data, taken from Jesus et al. (Ref. 6)].

son of the original waveguide with an isovelocity equivalent. Therefore, as a contribution to the general problem of acoustic tomography this paper presents the set of fundamental requirements for successful tomographic inversion of acoustic data in the context of travel-time-based shallow water tomography. This paper is organized as follows: Sec. II presents a brief theoretical review of linear inversion. This review is used in Sec. III to show, through simulations, that for deep water the number of independent arrivals is equal to the number of measured resolved travel times, while in shallow water the number of independent arrivals is much smaller than the number of actually measured resolved arrivals. The results of shallow water simulations are explained in Sec. IV through the comparison of the original acoustic waveguide with an isovelocity equivalent, and conclusions are drawn in Sec. V.

\section{LINEAR INVERSION: THEORETICAL BACKGROUND}

It can be shown on the basis of ray theory that the perturbation in travel time of an acoustic pulse can be written as $^{1,2}$

$$
\Delta \tau=\int_{\Gamma} \frac{d s}{c(z)}-\int_{\Gamma_{0}} \frac{d s}{c_{0}(z)},
$$

where $\Gamma$ and $\Gamma_{0}$ represent the eigenrays corresponding, respectively, to the perturbed and background sound-speed profiles $c(z)$ and $c_{0}(z)$. The background sound-speed profile $c_{0}(z)$ is considered to be known, for instance, from historical data. For small perturbations of sound speed $\delta c(z)=c(z)$ $-c_{0}(z) \ll c_{0}(z)$ one can take $\Gamma \approx \Gamma_{0}$, so the previous equation becomes

$$
\Delta \tau_{i}=\tau_{i}-\tau_{i}^{0}=\int_{\Gamma_{i}} \frac{d s}{c(z)}-\int_{\Gamma_{i}} \frac{d s}{c_{0}(z)} \approx-\int_{\Gamma_{i}} \frac{\delta c(z)}{c_{0}^{2}(z)} d s,
$$

where the integral is taken along the unperturbed eigenray $\Gamma_{i}$. The fundamental statement of this relationship is that a first-order perturbation in sound speed leads only to a first- order perturbation in travel time, while the path of the eigenray is not affected by this perturbation. In this sense $\Gamma_{i}$ corresponds to a stable eigenray and $\tau_{i}$ and $\tau_{i}^{0}$ can be considered as resolved travel times (or resolved arrivals). It is clear that the number of perturbations in travel time should be equal to the number of resolved eigenrays or, correspondingly, to the number of resolved arrivals. By "collecting" a set of T perturbations in travel time and representing the acoustic waveguide as a system composed of $L$ layers, one obtains the following linear system: ${ }^{2}$

$$
\mathbf{y}=\mathbf{E x}+\mathbf{n},
$$

where $\mathbf{y}=\left[\Delta \tau_{1} \Delta \tau_{2} \ldots \Delta \tau_{\mathrm{T}}\right]^{t}, \mathbf{x}=\left[\delta c_{1} \delta c_{2} \ldots \delta c_{\mathrm{L}}\right]^{t}$, each $\delta c_{j}$ is an average of $\delta c(z)$ in the $j$ th layer, and $\mathbf{n}$ represents the contribution of noise to the set of observations $\mathbf{y}$. Since the linear inversion will be tested with simulated data it will be considered in the following that there is a perfect match between both sides of the equation and the observations are fully deterministic (i.e., $\mathbf{n}=0$ ).

Matrix E, dimension $\mathrm{T} \times \mathrm{L}$, is called the "kernel matrix," the $\mathbf{e}_{i}$ of which have the following structure:

$$
\mathbf{e}_{i}=\left[\frac{\Delta s_{i 1}}{c_{01}^{2}} \frac{\Delta s_{i 2}}{c_{02}^{2}} \ldots \frac{\Delta s_{i \mathrm{~L}}}{c_{0 \mathrm{~L}}^{2}}\right],
$$

where $\Delta s_{i j}$ stands for the length of ray $i$ inside layer $j$ with $i=1,2, \ldots, \mathrm{T}$ and $j=1,2, \ldots, \mathrm{L}$. The choice of the number of layers $L$ can be done in many different ways. In general $L$ is made as large as possible and in practice it is often larger than T. Under this assumption of $L>T$, Eq. (3) consists of an underdetermined system of equations that has more unknowns than equations, and therefore has an infinite number of solutions. Formally, the columns of matrix $\mathbf{E}$ form a dependent set and, in practice, there is also no guarantee that $\mathrm{T}$ rows of $\mathbf{E}$ are linearly independent, which is equivalent to saying that $\mathbf{E}$ may be rank deficient. In terms of the underlying problem of time delays and sound-speed perturbations, rank deficiency means that not all resolved arrivals carry independent sound-speed information. Straight linear algebra tells us that such a system of equations has a solution $\mathbf{x}$, but that solution is not unique; that is to say that further information is needed to pick one among the possible solutions. The set of possible solutions are those that satisfy the system of equations

$$
\mathbf{E} \hat{\mathbf{x}}=\mathbf{p},
$$

where $\hat{\mathbf{x}}=\left[\mathbf{E}^{t} \mathbf{E}\right]^{-1} \mathbf{E}^{t} \mathbf{y}$ and therefore $\mathbf{p}$ is the projection of $\mathbf{y}$ onto the column space of $\mathbf{E}$. If such additional information is not available, the solution of Eq. (5) is the one that has minimum length. That solution is generally called the minimum norm solution and is given by the pseudoinverse

$$
\mathbf{x}^{\#}=\mathbf{E}^{\#} \mathbf{y} .
$$

The pseudoinverse $\mathbf{E}^{\#}$ is efficiently computed through the singular value decomposition ${ }^{7}$ (SVD) of matrix $\mathbf{E}, \mathbf{E}$ $=\mathbf{U S V}^{t}$, which provides a way of dealing with the rank of $\mathbf{E}$ by analysis of the singular spectra, $\sigma_{1}, \sigma_{2}, \ldots, \sigma_{\mathrm{T}}$, diagonal entries of $\mathbf{S}$, and further selection of the significant $\sigma_{i}$ in the SVD. However, such selection can not be done in a unique manner since it generally depends on the particular charac- 

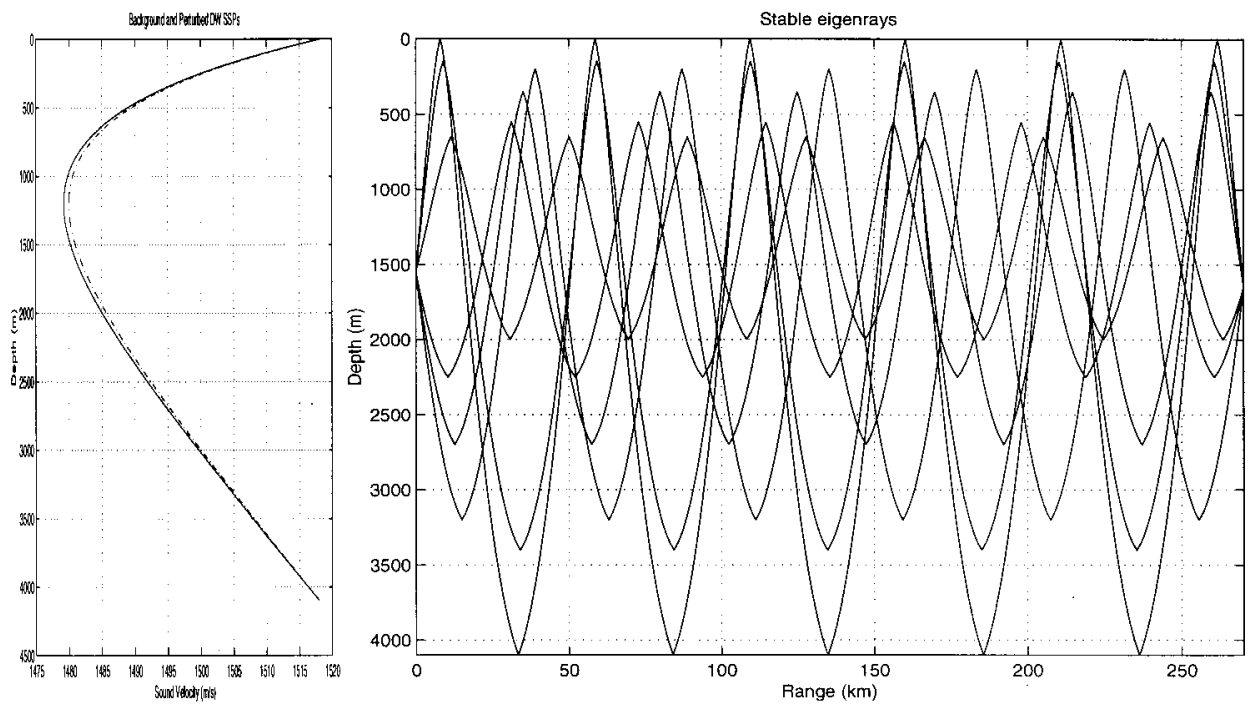

FIG. 2. Deep water test: Background $c_{0}(z)$ (dotted-dashed line) and perturbed $c(z)$ (continuous line) SSPs (left); stable eigenrays (right).

teristics of the problem. And even with the SVD solution being a minimum norm solution, nothing guarantees that such solution will be close to the searched solution, which is to say that minimizing $\left\|\mathbf{x}^{\#}\right\|$ does not imply the minimization of $\left\|\mathbf{x}^{\#}-\mathbf{x}\right\|$.

Finally, once the rank of the kernel matrix has been calculated, the minimum norm solution can be written as

$$
\mathbf{x}^{\#}=\mathbf{V}_{r} \mathbf{S}_{r}^{-1} \mathbf{U}_{r}^{t} \mathbf{y}
$$

where subscript $r=\operatorname{rank}(\mathbf{E})$, and denotes that matrices $\mathbf{V}$ and $\mathbf{U}$ are formed by their $r$ first singular vectors, and matrix $\mathbf{S}_{r}$ is square with its first (highest) singular values along the diagonal.

\section{SIMULATION TESTS}

Using the theoretical background presented in the previous section, travel-time-based tomographic inversion through ray-tracing simulations is tested to determine the number of independent arrivals in both deep and shallow water scenarios. For each scenario a background and a perturbed sound-speed profile (SSP) are chosen in order to obtain a negative perturbation of sound speed, which corresponds to positive perturbations in travel time. For each SSP a set of eigenrays is calculated and the set of stable eigenrays, resolved arrivals, and corresponding perturbations in travel time are determined. The kernel matrix, E, is constructed with the stable eigenrays and then the inverse solution is calculated from its SVD. When dealing with real data the number of independent eigenrays $N$ (which is the same as the number of independent arrivals) can be estimated by using statistical criteria. ${ }^{6,8}$ Since the test case presented here is fully deterministic, an alternative method for estimating the rank of matrix $\mathbf{E}$ is proposed. That method takes advantage of the structure of the inverse solution based on the SVD of the kernel matrix, which was discussed in the previous section, and introduces the following functional:

$$
E(i)=\frac{\left\|\mathbf{x}_{i}^{\#}-\mathbf{x}\right\|^{2}}{\left\|\mathbf{x}_{i}^{\#}\right\|^{2}},
$$

where $\mathbf{x}_{i}^{\#}$ is the inverse solution obtained from Eq. (7) and calculated with the first $i$ singular values. The "real" perturbation $\mathbf{x}$ is calculated from $\delta c(z)$ (which is known in our simulated case) according to the adopted depth discretization. Using the functional $E(i)$ one can obtain the following estimator of the number of independent arrivals $N$ :

$$
\hat{N}=\underset{i}{\arg \left\{\min _{i} E(i)\right\}}
$$

The minimum of $E(i)$ does not have to be a minimum in the conventional sense since solutions with $N=1$ or $N=\mathrm{T}$ will also be admitted. If $N=\mathrm{T}$ (which should not be surprising) the natural conclusion is that all resolved arrivals are independent and therefore they all contribute with independent information to the tomographic inversion. However, if $N$ $<\mathrm{T}$ (and from ray tracing there is no apparent reason for this to be so), then the unexpected conclusion is that only $N$ of $\mathrm{T}$ resolved arrivals are independent, and the remaining $N-\mathrm{T}$ convey redundant information. Those redundant arrivals will not contribute with additional information to the tomographic inversion. It will be shown in the following subsections that in deep water one obtains the "expected" conclusion $(N=\mathrm{T})$, while in shallow water part of the resolved arrivals are redundant, i.e., $N<\mathrm{T}$.

\section{A. Deep water test}

The well-known analytical expression for the Munk velocity profile was used to generate the SSPs (see left panel of Fig. 2). Following the geometry of a real experiment ${ }^{4}$ the acoustic source and the receiver depths are $z_{s}=1500$ and $z_{r}$ $=1650 \mathrm{~m}$, respectively, the depth of the acoustic waveguide is $D=4100 \mathrm{~m}$, and the distance separating the source and the receiver is $R=270 \mathrm{~km}$. The asymmetry $z_{s} \neq z_{r}$ is intentional. In fact, as discussed by Munk et al., ${ }^{2}$ by locating both source and receiver at the same depth one gets symmetric eigenrays, with turning points at the same depths. Therefore, those eigenrays sample the ocean in the same way and constitute a preliminary source of redundancy in the kernel matrix, which should be avoided. After eigenray ray tracing for the background and the perturbed SSPs, a set of five RR stable eigen- 


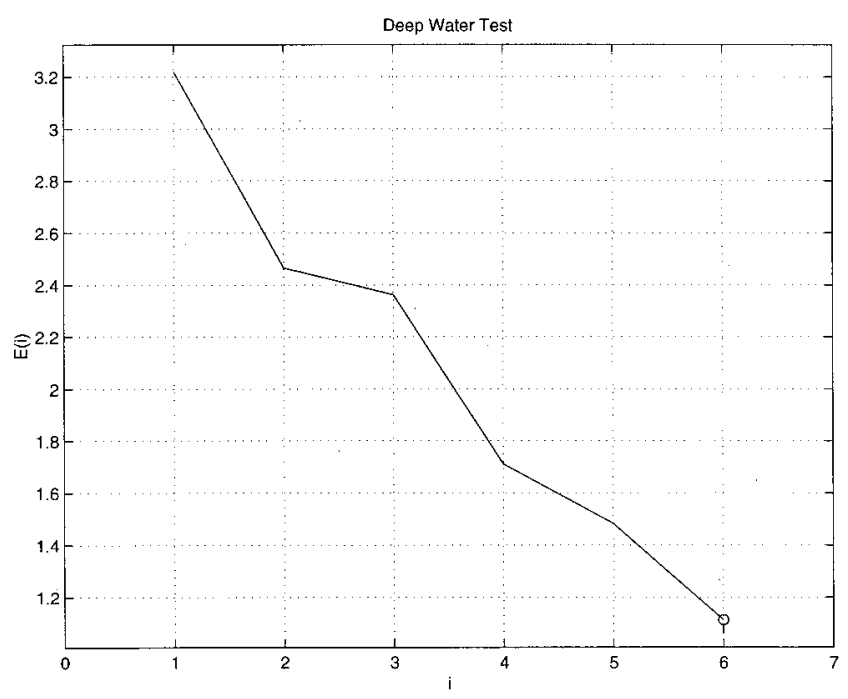

FIG. 3. Deep water test: Estimation of independent arrivals; the projection of the minimum [Eq. (8)] onto the horizontal axis indicates the number of independent arrivals $N$.

rays and one surface-reflected-bottom-reflected (SRBR) stable eigenray were found (see right panel of Fig. 2). The reflected eigenray should be considered in a somehow formal way (in fact this is the only eigenray that spans the entire water column) since in real conditions the amplitude of SRBR eigenrays is difficult to detect over the level of environmental noise. ${ }^{9}$ Using Eq. (8) it can be found that $N=6$ (see Fig. 3). From this result it can be concluded that all the resolved arrivals are independent and this is the "expected" conclusion.

\section{B. Shallow water test}

The shallow water background SSP for this test corresponds to the mean profile from conductivity, temperature, depth (CTD) data used in Jesus et al. $;^{6}$ a particular profile from the same data was considered to be representative of the perturbed SSP (see left panel of Fig. 4). The geometry of propagation was taken also from that reference, with the acoustic source at depth $z_{s}=90 \mathrm{~m}$, the hydrophone at depth $z_{r}=115 \mathrm{~m}$ and range $R=5.6 \mathrm{~km}$, and the total depth of the waveguide being $D=135 \mathrm{~m}$. As in the deep water test, the asymmetry $z_{s} \neq z_{r}$ avoids the redundancy of symmetric eigenrays with equal turning depths. From ray tracing it can be found that all eigenrays are of RBR or SRBR types (see Fig. 5). The RBR eigenrays [Fig. 5(a)] are not stable (see left box of right panel of Fig. 4) and therefore they can not be used in the tomographic inversion. The SRBR eigenrays [Fig. 5(b)] are stable and "clustered" in quadruplets and are, therefore, suitable for inversion purposes (see right box on right panel of Fig. 4). In general, the clustering of arrivals depends on the particular characteristics of the waveguide geometry and associated SSP. For the shallow water environment and SSP of this test one can remark that each quadruplet contains the arrival times $\tau$, ordered according to the general sequence,

$$
\left(\tau_{2 m-1}^{+}, \tau_{2 m}^{+}, \tau_{2 m}^{-}, \tau_{2 m+1}^{-}\right),
$$

where the index of each $\tau$ represents the number of reflections on the surface or bottom of the corresponding eigenray, a "+" or a "-" sign indicates whether that eigenray was launched toward the surface or toward the bottom, respectively. To calculate the kernel matrix an homogeneous layer grid was introduced. Each layer has a thickness $\Delta z=4 \mathrm{~m}$, which is four times more than the spatial resolution of the discretized sound-speed profile. The depth of every layer interface was coincident with every fourth depth of the discretized sound speed. To simplify the calculations, additional interfaces were added at depths $z_{s}, z_{r}$, and $D$, which were not included in the homogeneous grid. Thus a total of 36 layers was used to calculate the kernel matrix. The sound speed for each layer was the average of the discretized sound speeds contained within the layer. The functional $E(i)$ was calculated considering a total of 20 resolved arrivals. However, its minimum is reached at $N=4$ (see Fig. 6), which indicates that only 4 of the 20 resolved arrivals are independent, while the other 16 are redundant. It should be remarked that this result is in agreement with a statistical estimation of uncorrelated paths presented in Jesus et al. ${ }^{6}$ It is clear that

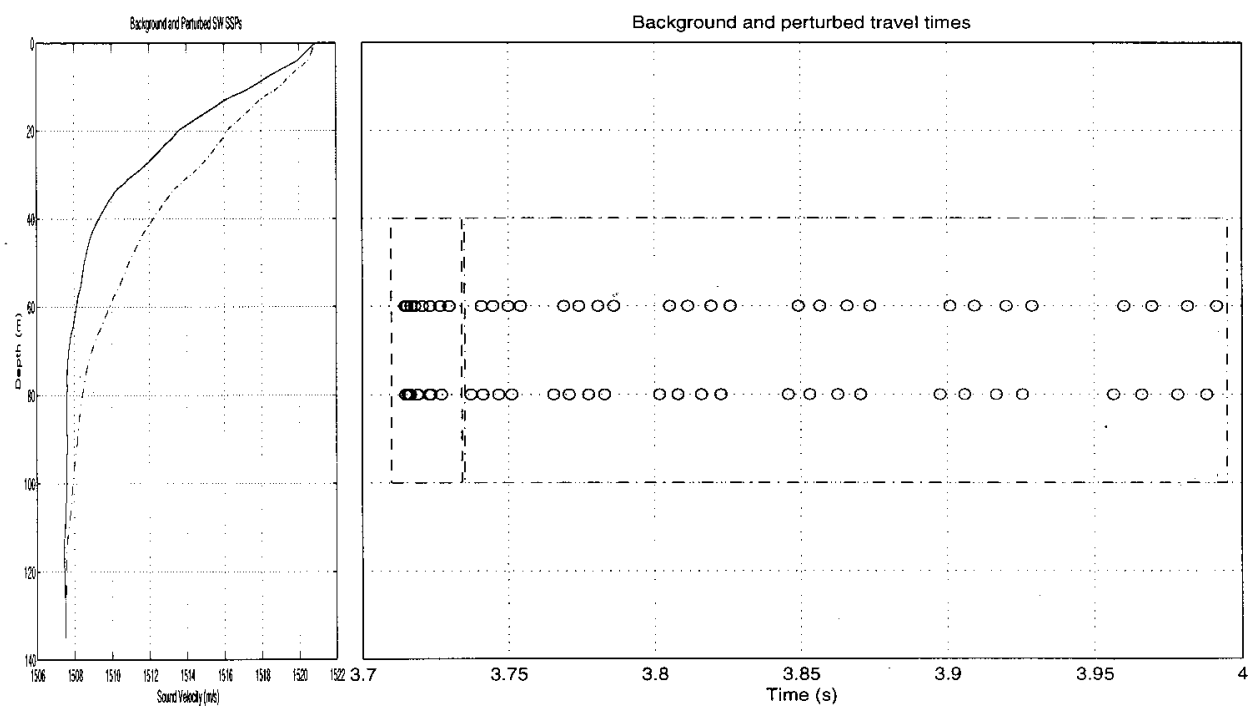

FIG. 4. Shallow water test: Background $c_{0}(z)$ (dotted-dashed line) and perturbed $c(z)$ (continuous line) SSPs (left); background $\tau_{0}$ (lower sequence) and $\tau$ (upper sequence) travel times (right), left box indicates unstable arrivals, right box indicates resolved arrivals. 

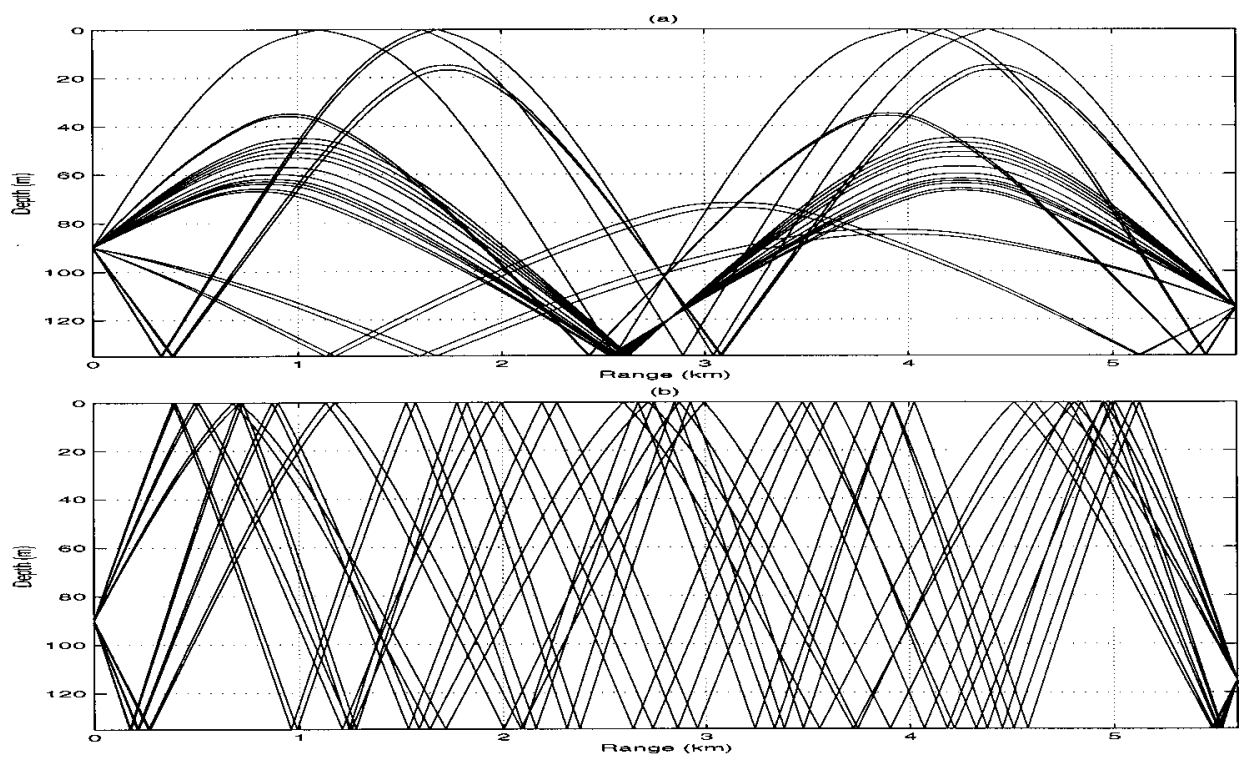

FIG. 5. Rays of propagation for unstable arrivals (a) and stable arrivals (b). (For simplicity only the first three quadruplets are shown.) the result depends deeply on the particular structure of the kernel matrix, which will be discussed in the following section.

\section{DISCUSSION}

The simulation results obtained in the previous section show that the number of independent arrivals (and therefore, of independent eigenrays) can be much lower than the number of resolved arrivals. It follows from those results that there are fundamental physical limitations to the number of independent parameters available for travel-time tomography. However, the general understanding of the simulation results still remains incomplete because those results only say how many of the eigenrays are independent, but they do not say which are the independent eigenrays and the reason for being so. Intuitively it seems reasonable to admit that each set of eigenrays, corresponding to a particular quadruplet, are independent, and therefore, that each of those eigen-

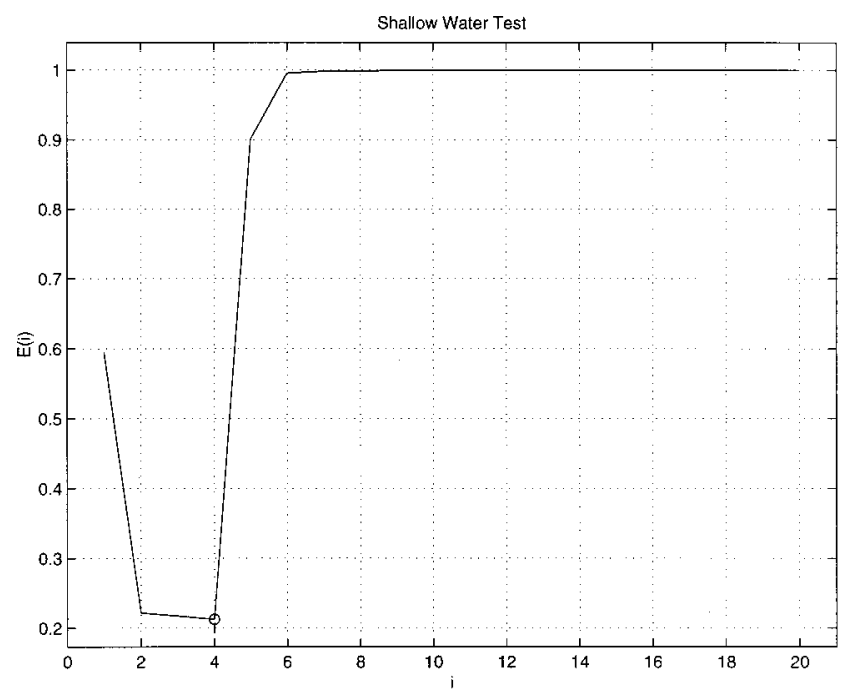

FIG. 6. Shallow water test: Estimation of independent arrivals; the projection of the minimum [Eq. (8)] onto the horizontal axis indicates the number of independent arrivals $N$. rays contains a "piece" of independent information. In mathematical terms this assumption states not only that rank $(\mathbf{E})=4$, but also that for a given quadruplet $q$ the corresponding four rows in $\mathbf{E}$ are linearly independent, and can be used to calculate the four rows of any other quadruplet. However, within the context of ray theory there is not a clear explanation to support this assumption. In part this is due to the fact that, for a generic sound-speed profile $c_{0}(z)$, one can not derive explicit analytic expressions for each row $\mathbf{e}_{i}$ of the kernel matrix, thus "hiding" any possible dependence between different sets of rows. In general, for a shallow water waveguide, one can expect that most of the SRBR eigenrays are characterized by steep launching angles and by a significant number of reflections on both surface and bottom. As the number of reflections increases, the shape of the SRBR eigenrays tends to be closer to straight lines. Therefore, for a waveguide geometry like the one discussed in the shallow water test, but with an equivalent_constant—sound-speed profile, the isovelocity kernel matrix can provide a reasonable approximation to the original matrix E. Moreover, for a constant $c_{0}$, each row of $E$ can be explicitly calculated, making it possible to understand which eigenrays are the independent ones. Those results can provide fundamental knowledge related to the structure of the original kernel matrix, and thus provide an answer to the questions discussed in the beginning of this section.

In general, an SRBR eigenray launched to the surface can arrive at the hydrophone after being reflected an odd number of times $2 m-1$, or after being reflected an even number of times $2 m$, where $m$ can take the values $1,2, \ldots$. The same kind of reasoning can be applied to an SRBR eigenray being launched to the bottom. Thus for a fixed $m$, there are four types of eigenrays connecting source and receiver. In the isovelocity case the launching angles of these four eigenrays can be derived by inspection and are given by

$$
\tan \theta_{2 m-1}^{+}=\frac{(2 m-2) D+z_{s}+z_{r}}{R}
$$




$$
\begin{aligned}
& \tan \theta_{2 m}^{+}=\frac{2 m D+z_{s}-z_{r}}{R}, \\
& \tan \theta_{2 m-1}^{-}=\frac{2 m D-z_{s}-z_{r}}{R}, \\
& \tan \theta_{2 m}^{-}=\frac{2 m D-z_{s}+z_{r}}{R},
\end{aligned}
$$

where the convention of the "+" or a "-" sign was already introduced in the discussion of the shallow water test. The number of total reflections is given by the index of each $\theta$. There is no practical sense in calculating the $\theta_{m}$ for large values of $m$ because the contribution of a particular eigenray to the pressure field decreases as the number of reflections increases. Furthermore, the arrival times correspond to

$$
\tau_{m}^{+/-}=\frac{R}{c_{0} \cos \theta_{m}^{+l-}} .
$$

For an isovelocity SSP the clustering of arrivals depends mainly on the particular values of $z_{s}, z_{r}, D$, and $R$. However, by taking the values used in the shallow water test, and taking $c_{0}=1510 \mathrm{~m} / \mathrm{s}$, it can be found that the set of four arrivals will be ordered again according to the general sequence Eq. (10). For the sake of simplicity let us consider further that the linear inversion is performed with a set of $q$ quadruplets, so $T=4 q$. A simple choice of the layer system consists in selecting a homogeneous grid composed of $L$ layers, each with a thickness $\Delta z=D / L$. The layer thickness will be taken sufficiently small to separate the source and the receiver with at least a single layer, i.e., the layer indexes will obey the following order:

$$
j=1,2, \ldots, \mathrm{L}=1,2, \ldots, \mathrm{S}, \mathrm{S}+1, \ldots, \mathrm{R}, \mathrm{R}+1, \ldots, \mathrm{L} .
$$

The indexes $\mathrm{S}$ and $\mathrm{R}$ correspond to the integer parts of $z_{s} / \Delta z$ and $z_{r} / \Delta z$, respectively. Furthermore, for the traveltime sequence given by Eq. (10) the isovelocity kernel matrix can be written as

$$
\begin{aligned}
\mathbf{E}=\left[\begin{array}{c}
\mathbf{e}_{1} \\
\mathbf{e}_{2} \\
\mathbf{e}_{3} \\
\mathbf{e}_{4} \\
\mathbf{e}_{5} \\
\vdots \\
\mathbf{e}_{\mathrm{T}}
\end{array}\right]=\left[\begin{array}{c}
{\left[\Delta s_{11} \Delta s_{12} \cdots \Delta s_{1 \mathrm{~L}}\right] / c_{0}^{2}} \\
{\left[\Delta s_{21} \Delta s_{22} \cdots \Delta s_{2 \mathrm{~L}}\right] / c_{0}^{2}} \\
{\left[\Delta s_{31} \Delta s_{32} \cdots \Delta s_{3 \mathrm{~L}}\right] / c_{0}^{2}} \\
{\left[\Delta s_{41} s_{42} \cdots \Delta s_{4 \mathrm{~L}}\right] / c_{0}^{2}} \\
{\left[\Delta s_{51} \Delta s_{52} \cdots \Delta s_{5 \mathrm{~L}}\right] / c_{0}^{2}} \\
\vdots \\
{\left[\Delta s_{\mathrm{T} 1} s_{\mathrm{T} 2} \cdots \Delta s_{\mathrm{TL}}\right] / c_{0}^{2}}
\end{array}\right] \\
=\left[\begin{array}{c}
\alpha_{2 \mathrm{M}-1}^{+} \times \mathbf{e}_{2 \mathrm{M}-1}^{+} \\
\alpha_{2 \mathrm{M}}^{+} \times \mathbf{e}_{2 \mathrm{M}}^{+} \\
\alpha_{2 \mathrm{M}}^{-} \times \mathbf{e}_{2 \mathrm{M}}^{-} \\
\alpha_{2 \mathrm{M}+1}^{-} \times \mathbf{e}_{2 \mathrm{M}+1}^{-} \\
\alpha_{2 \mathrm{M}+1}^{+} \times \mathbf{e}_{2 \mathrm{M}+1}^{+} \\
\vdots \\
\alpha_{2 \mathrm{M}+2 q-1}^{-} \times \mathbf{e}_{2 \mathrm{M}+2 q-1}^{-}
\end{array}\right],
\end{aligned}
$$

where $\alpha_{m}^{+/-}=\left(c_{0}^{2} \sin \theta_{m}^{+/-}\right)^{-1}$, and the index $2 \mathrm{M}-1$ represents the number of even reflections of the first eigenray within the first quadruplet. The rows $\mathbf{e}_{m}^{+/-}$are given by

$$
\begin{aligned}
\mathbf{e}_{2 m-1}^{+}= & {[2 m \Delta z 2 m \Delta z \ldots(2 m-\mathrm{S}) \Delta z} \\
& +z_{s}(2 m-1) \Delta z \ldots(2 m-1-\mathrm{R}) \Delta z \\
& \left.+z_{r}(2 m-2) \Delta z \ldots(2 m-2) \Delta z\right], \\
\mathbf{e}_{2 m}^{+}=[ & 2 m \Delta z 2 m \Delta z \ldots(2 m-\mathrm{S}) \Delta z+z_{s}(2 m-1) \Delta z \ldots \\
& \left.(2 m-1+\mathrm{R}) \Delta z-z_{r} 2 m \Delta z \ldots 2 m \Delta z\right], \\
\mathbf{e}_{2 m}^{-}=[ & 2 m \Delta z 2 m \Delta z \ldots(2 m+\mathrm{S}) \Delta z \\
- & z_{s}(2 m+1) \Delta z \ldots(2 m+1-\mathrm{R}) \Delta z \\
+ & \left.z_{r} 2 m \Delta z \ldots 2 m \Delta z\right], \\
\mathbf{e}_{2 m+1}^{-}= & {[2 m \Delta z 2 m \Delta z \ldots(2 m+\mathrm{S}) \Delta z} \\
& -z_{s}(2 m+1) \Delta z \ldots(2 m+1+\mathrm{R}) \Delta z \\
& \left.-z_{r}(2 m+2) \Delta z \ldots(2 m+2) \Delta z\right] .
\end{aligned}
$$

It follows from the previous set of equations that the rows $\mathbf{e}_{m}^{+/-}$can be calculated recursively, through the relationship

$$
\mathbf{e}_{2 m+1}^{+l-}-\mathbf{e}_{2 m-1}^{+l-}=\mathbf{e}_{2 m+2}^{+/-}-\mathbf{e}_{2 m}^{+/-}=[2 \Delta z 2 \Delta z \ldots 2 \Delta z] .
$$

As shown by Eqs. (15), every four rows $\mathbf{e}_{i}$ corresponding to a given quadruplet are independent. Furthermore, since $\alpha_{i}$ is a common factor to all the components of each row $\mathbf{e}_{i}$, the set Eqs. (16) indicates the linear dependence between each pair of rows $\mathbf{e}_{i}$ and $\mathbf{e}_{i+4}$. In this way, the previous analysis of the isovelocity kernel matrix indicates not only how many of the eigenrays are independent [since the analysis shows that rank $(\mathbf{E})=4$ ], but indicates also in detail which are the independent eigenrays. For the case of a more generic soundspeed profile $c_{0}(z)$, as the number of reflections increases, one notes that the slope of each SRBR eigenray approaches a constant, given by the slope of the launching angle $\tan \theta$. Also significant is that the length of a single eigenray crossing a particular layer approaches the ratio $\Delta z / \sin \theta$. In this way, the general structure of Eqs. (15) suggests that, for the shallow water test, each row of $\mathbf{E}$ can be approximated as

$$
\mathbf{e}_{i} \approx \alpha_{i} \times\left[\mathrm{M}_{i 1} \frac{\Delta z}{c_{01}^{2}} \mathrm{M}_{i 2} \frac{\Delta z}{c_{02}^{2}} \cdots \mathrm{M}_{i \mathrm{~L}} \frac{\Delta z}{c_{0 \mathrm{~L}}^{2}}\right],
$$

where $\alpha_{i}=\left(\sin \theta_{i}\right)^{-1}$ and $\mathrm{M}_{i j}$ represents the number of times that the eigenray $i$ crosses the layer $j$. Through further analogy the set Eqs. (15) guarantees that there are at least four different types of row components (since the layer thickness is not a common factor), and that guarantees the linear independence of those four rows $\mathbf{e}_{i}$, corresponding to a particular quadruplet. The analogy to Eqs. (15) allows one to note also that

$\mathbf{e}_{i+4} \approx \alpha_{i+4} \times\left[\left(\mathrm{M}_{i 1}+2\right) \frac{\Delta z}{c_{01}^{2}}\left(\mathrm{M}_{i 2}+2\right) \frac{\Delta z}{c_{02}^{2}} \cdots\left(\mathrm{M}_{i \mathrm{~L}}+2\right) \frac{\Delta z}{c_{0 \mathrm{~L}}^{2}}\right]$,

which brings back the linear dependence between each pair of rows $\mathbf{e}_{i}$ and $\mathbf{e}_{i+4}$. Thus the analysis of the isovelocity kernel matrix, and its analogy to the kernel matrix of the original shallow water waveguide, provide a full understanding of the results of the shallow water test. 


\section{CONCLUSIONS}

On the basis of this analysis the following conclusions can be drawn: (1) in the context of travel-time-based shallow water tomography it is of fundamental importance to determine the number of independent resolved arrivals; (2) with real data the estimation of independent arrivals can be done through statistical tests, while in simulations the estimation can be performed by comparison of the inverse and expected solution; (3) it can be shown through ray-tracing simulation and under the condition of placing the source and the receiver at different depths, that in deep water the number of independent arrivals is equal to the number of resolved arrivals; corresponding simulations in shallow water reveal that the number of independent arrivals is much smaller than the number of actually measured-resolved-arrivals; (4) finally, the problem of travel-time redundancy in the shallow water waveguide is fully explained through the detailed analysis of the kernel matrix of an equivalent isovelocity waveguide, where the rows of the isovelocity matrix show a fundamental rank deficiency of the kernel matrix associated with the original shallow water waveguide.

\section{ACKNOWLEDGMENTS}

The authors deeply want to thank the reviewers for the interest they have shown in the discussion of the material presented in this publication. Their detailed remarks concerning some incomplete aspects of the material presented in the first version of the manuscript undoubtedly guided the authors to develop a fundamental improvement of that material.

${ }^{1}$ W. Munk and C. Wunsch, "Ocean acoustic tomography: A scheme for large scale monitoring," Deep-Sea Res., Part A 26, 123-161 (1979).

${ }^{2}$ W. Munk, P. Worcester, and C. Wunsch, "Ocean acoustic tomography," Cambridge Monographs on Mechanics, New York, 1995.

${ }^{3}$ Y. Stéphan and S. Thiria, "Neural inversions for ocean acoustic tomography," in Inverse Problems in Engineering Mechanics, edited by Bui Tanaka et al. (Balkema, 1994).

${ }^{4}$ S. Prasanna, Y. K. Somayajulu, T. V. Ramana, G. S. Navelkar, A. K. Saran, A. M. Almeida, and C. S. Murty, "Preliminary results of an acoustic tomography experiment (ATE-93) in the eastern Arabian Sea," Proceedings of the 2nd European Conference on Underwater Acoustics, edited by L. Bjorno, 1994.

${ }^{5}$ C-S. Chiu, J. H. Miller, and J. F. Lynch, "Inverse techniques for coastal acoustic tomography,"' in Environmental Acoustics, edited by D. Lee and M. Schultz (World Scientific, Singapore, 1994).

${ }^{6}$ S. M. Jesus, M. B. Porter, Y. Stephan, X. Démoulin, O. Rodríguez, and E. Coelho, "Single hydrophone source localization," IEEE J. Ocean Eng. 25(3), 337-346 (2000).

${ }^{7}$ W. Menke, Geophysical Data Analysis: Discrete Inverse Theory (Academic, San Diego, 1989).

${ }^{8}$ M. Wax and T. Kailath, "Detection of signals by information theoretic criteria," IEEE Trans. Acoust., Speech, Signal Process. 33, No. 2, $387-$ 392 (1985).

${ }^{9}$ I. Tolstoy and C. S. Clay, Ocean Acoustics, Theory and Experiment in Underwater Sound (AIP, New York, 1987). 